\section{To embrace doping in sport is absurd}

I find your discussion of performance-enhancing drugs (Nature 487, 287-289; 2012) disrespectful to the millions of elite and sub-elite athletes who rely solely on training to reach their goals. In reality, most of these drugs do not create "superhuman athletes" but allow athletes to take short cuts in training or to recover faster from injury.

The sport of running has been plagued by doping for years, and a slew of positive tests has been reported in recent weeks. But to suggest that doping should be embraced because drug-testing efforts are a losing battle is absurd. For every performance-enhancing-drug pragmatist, there are many who would rather see stricter testing regimens and harsher penalties for offenders.

Science does hold promise for future athletes wanting to break today's barriers, but the realms of exercise science, sports psychology and nutrition are likely to have more bearing than pharmaceuticals and genetics (Nature 487, 297; 2012). Ryan Purcell Emory University, Atlanta, Georgia, USA. ryan.purcell@emory.edu

\section{Misconduct rule is not retroactive}

Octavian Voiculescu is concerned that the latest regulations of the Romanian National Ethics Council might be used retroactively, which would be unconstitutional (Nature 486, 34; 2012). As president of Romania's National Authority for Scientific Research that helped to introduce these regulations in 2011, I would like to stress that this is not the case: the regulations are not implemented retroactively.

The regulatory changes include a provision to terminate automatically the employment contracts of academics or researchers found guilty of "grave misconduct" - plagiarism, falsification of scientific data or false statements in grant applications.

The non-retroactive legal principle means that no misconduct committed before the legislation came into effect can be penalized on the basis of that legislation. This principle has been strictly observed under my administration.

It is incorrect to interpret the non-retroactive principle as meaning that sanctions cannot remove rights or privileges that were in operation before the legislation came into effect, with people losing their jobs only if they were appointed after 2011.

It is not the date of employment that matters, but the date on which the illegal action was undertaken: if the action was perpetrated before 2011, it cannot be sanctioned; if it happened afterwards, it can.

Dragoş Ciuparu Petroleum-Gas University of Ploieşti, Romania. dciuparu@upg-ploiesti.ro

\section{Put patients and researchers in touch}

As Research Network leader at the UK Alzheimer's Society, I can vouch that the value of the biomedical research community engaging with patients and carers acts both ways (Nature 487, 7; 2012).

Research supported by the Public and Patient Involvement programme of the UK Alzheimer's Society couples scientific and clinical expertise with the insight of people who are affected directly or indirectly by dementia (www.alzheimers. org.uk/researchnetwork).These people convey their research priorities to us; we offer them an equal presence and vote at funding panels.

For every grant we commission, we assign three carers, former carers or people with dementia to provide an informal monitoring group who visit the researchers once or twice a year. These volunteers encourage the research team by sharing their own experiences, and help us to improve our protocol, patient communication and recruitment methods.

The patients and carers learn more about the complexity, challenges and timescales of biomedical research. The researchers come to realize that dementia is about much more than memory loss, and that sufferers are still human beings.

This two-way communication motivates patients and carers to fund-raise and campaign for the Alzheimer's Society. Since 1999, we have awarded more than $£ 15$ million (US\$24 million) to these research partnerships. Matt Murray Alzheimer's Society, London, UK.

matt.murray@alzheimers.org.uk

\section{Alarming shift away from sharing results}

There have been worrying attempts in the past year to tamper with the sharing of scientific research results. Each seems indicative of a shift away from the classical principles of science.

There was the review of two $\mathrm{H} 5 \mathrm{~N} 1$ avian influenza virus studies in ferrets by the US National Science Advisory Board for Biosecurity in December 2011. The board initially recommended that the published studies should "not include the methodological and other details that could enable replication of the experiments by those who would seek to do harm" (see go.nature. com/nywkdy). Fortunately, this attempted ban was later withdrawn.

In two disturbing examples of publication irregularities, a meta-review of biomedical papers in leading journals revealed that key primary data are not always made publicly available (A. A. Alsheik-Ali et al. PLoS ONE 6, e24357; 2011); and
Bernardo Huberman alerted the community to the practice of using non-disclosed data from private sources (Nature 482, 308; 2012).

In December 2011 a bill was proposed to the US Congress to reverse the National Institutes of Health policy that all taxpayerfunded research should be freely accessible online (see go.nature.com/uvj681). The bill's proponents later withdrew their support, but legislative action would have severely limited the diffusion of scientific knowledge.

Science should be available for evaluation by other scientists and for public scrutiny, just as it has been since Galileo's time. It should not be heading for epistemological suicide as a result of vested interests or a creeping loss of awareness of the theory of knowledge.

Giovanni Boniolo University of Milan and European Institute of Oncology, Milan, Italy. Thomas Vaccari FIRC Institute of Molecular Oncology (IFOM), Milan, Italy.

thomas.vaccari@ifom.eu

\section{Time for a Higgs metaphor upgrade}

I liked your sidebar 'What is the Higgs?' (Nature 487, 147-148; 2012). This offers the first hint that there may be new explanations beyond the standard metaphor that it "endows particles with mass". After reading words to that effect in so many news articles that simply leave it at that, this scientifically literate layman is ready for a metaphor upgrade.

If this turns out to be 'the end' for theoretical physicists, maybe they could find gainful employment working with science writers to develop metaphors that match the levels of scientific and mathematical sophistication of a wider range of target audiences.

Charles Packer Washington DC, USA.mailbox@cpacker.org 